\title{
QMSCC Calculations on Thermal Quenching of Model Phosphor Systems
}

\author{
K. C. BLEIJENBERG AND G. BLASSE \\ Solid State Chemistry Department, Physical Laboratory, State University, \\ P.O. Box 80.000, 3508 TA Utrecht, The Netherlands
}

Received June 15, 1978

\begin{abstract}
Quantum mechanical single configurational coordinate (QMSCC) calculations have been performed on model phosphor systems. Variation of the QMSCC parameters provides information about the influence of these parameters on the temperature dependence of the luminescence efficiency. The results of the calculations are correlated with experimental results reported in the literature.
\end{abstract}

\section{Introduction}

A few years ago Struck and Fonger (1) proposed a quantitative method to describe the radiative and non-radiative relaxation from excited states using a quantum mechanical single configurational coordinate (QMSCC) model. Parameters for this model are depicted in Fig. 1. In this figure the horizontal coordinate is the single configurational coordinate $Q$, the vertical coordinate is the total energy $E$ of the system. The parabolas $u$ and $v$ are the potential energy wells of the electronic ground state and the relevant excited state, respectively. The $u$ and $v$ parabola quantum numbers, wavefunctions, and phonon energies are called $n, u_{n}, \hbar \omega_{u}$ and $m, v_{m}, \hbar \omega_{v}$, respectively. $a_{u v}$ measures the FranckCondon (FC) offset and is expressed in terms of $S_{u}$ and $S_{v}: a_{u v}^{2}=2\left(S_{u}+S_{v}\right) . S_{u} \cdot \hbar \omega_{u}$ and $S_{v} \cdot \hbar \omega_{v}$ represent the relaxation energies after emission and absorption, respectively. The QMSCC model is taken in the thermal Condon approximation; i.e., the initial vibrational states are in thermal equilibrium and the $v_{m} \rightarrow u_{n}$ rate is proportional to the squared overlap integral $\left\langle u_{n} \mid v_{m}\right\rangle^{2}(2)$. These overlap integrals are evaluated using the recursion formulas given by Manneback (3),

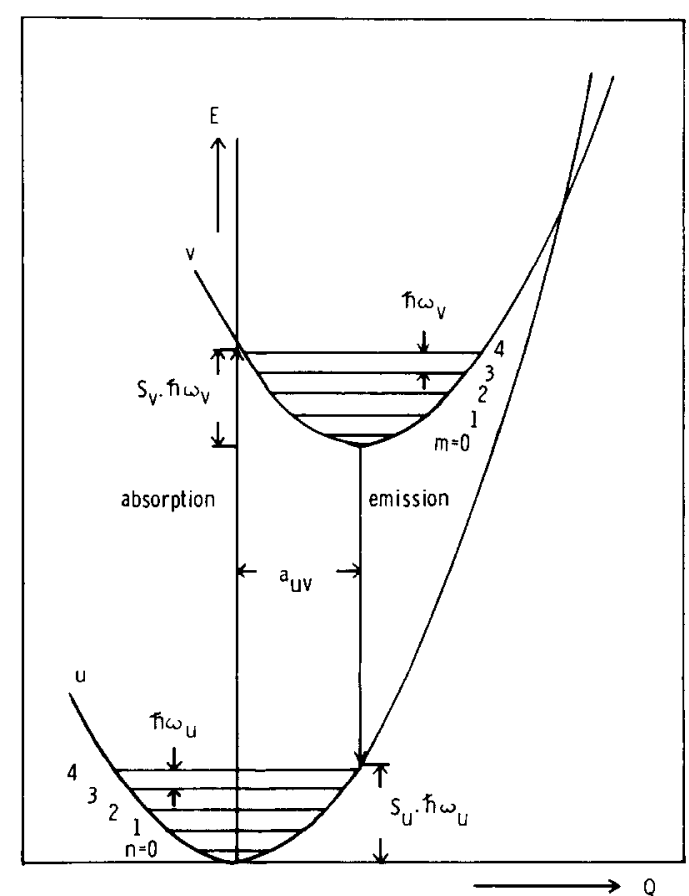

FIG. 1. Single configurational coordinate diagram. For explanation of the sym: sls see text. 
offering the possibility of a quantitative treatment of radiative and non-radiative processes.

Struck and Fonger applied QMSCC-calculations to the luminescence properties of ruby and emerald (4). Tyner and Drickamer used QMSCC-calculations to describe the temperature quenching of luminescence in tungstates, molybdates and $\mathrm{Eu}^{2+}$-activated phosphors $(5,6)$.

In this paper we will discuss QMSCC calculations that have been performed on model phosphors in order to study the influence, of variations of the configurational coordinate model parameters on the thermal quenching temperature and the efficiency of the luminescence.

\section{Results}

Starting point for our calculations was a hypothetical, red emitting phosphor. The emission spectrum and the absorption spectrum (Fig. 2) are due to radiative transitions within a two-level electronic scheme, and can be explained in terms of a two-parabola SCC diagram (Fig. 1). At higher temperatures non-radiative $v \rightarrow u$ relaxation becomes predominant resulting in luminescence quenching. For $v \rightarrow u$ transitions Struck and
Fonger describe the radiative and non-radiative transition probabilities $R$ and $N$ as follows:

$$
R=\sum_{p_{U}=-\infty}^{+\infty} R_{p_{U v}}=R_{u v} \sum_{p_{U}=-\infty}^{+\infty} U_{p_{U}}=R_{u v}
$$

$N=N_{p_{U v}}=N_{u v} U_{p_{U}}$

$R_{u v}$ and $N_{u v}$ are constants from the electronic part of the transition integral, $R_{p \text { vo }}$ and $N_{p \cup v}$ are summations of $v_{m} \rightarrow u_{n}$ transition rates for all $m$ and $n$ satisfying $m-n=$ $p_{U}$. (This holds for the linear coupling case. In the general case; i.e., $\hbar \omega_{u} \neq \hbar \omega_{v}, p_{U}$ is defined as the integer that approximates $n-$ $\left(\hbar \omega_{v} / \hbar \omega_{u}\right) m$.) In order to obtain the total radiative rate the $R_{p_{U v}}$ rate must be summed over all values of $p_{U}$. For non-radiative transitions only resonant states contribute to the total non-radiative rate. In resonance $p_{U}$ is defined as $E_{z p} / \hbar \omega_{u}$, where $E_{z p}$ is the zero phonon energy (the energy interval between $v_{0}$ and $\left.u_{0}\right)$. $U_{p_{U}}$ gives the temperaturedependent shape of the $v \rightarrow u$ optical band and the temperature dependence of the nonradiative rate. In the linear coupling case, i.e. $\hbar \omega_{v}=\hbar \omega_{u}$, the expression for $U_{p_{U}}$ reads:

$$
U_{p_{U}}=\sum_{m=m_{0}}^{\infty}\left(1-r_{v}\right) r_{v}^{m}\left\langle u_{p_{u}+m} \mid v_{m}\right\rangle^{2}
$$

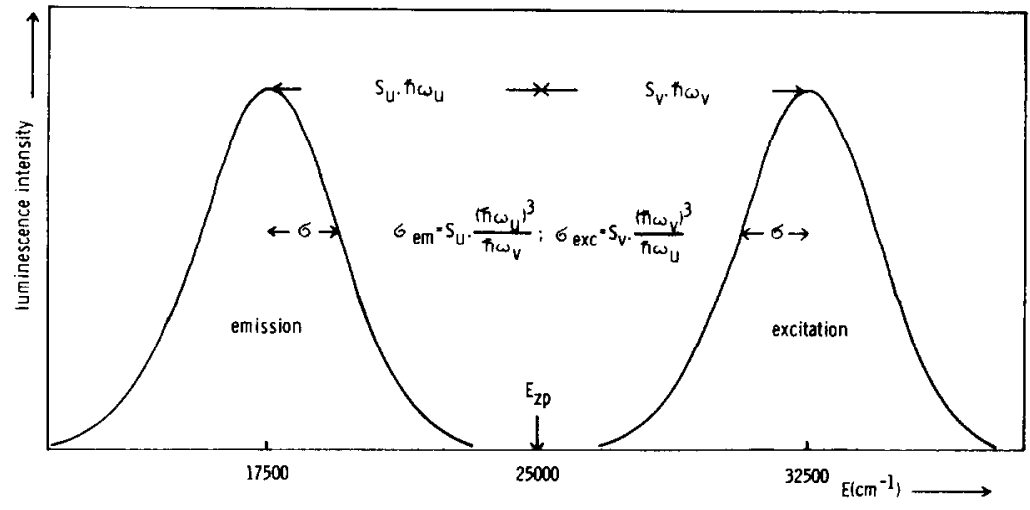

FIG. 2. Emission and absorption spectrum of the hypothetical phosphor at $0 \mathrm{~K} . \sigma_{\text {em }}$ and $\sigma_{\text {exc }}$ represent half the width of the emission and the excitation band at the intensity where the luminescence has decreased to $1 / e^{1 / 2}$ times its maximum value, at $0 \mathrm{~K}$. 
where $r_{v}=\exp \left(-\hbar \omega_{v} / k T\right)$ is the Boltzmann factor between adjacent initial vibrational states $v_{m},\left\langle u_{p_{u}+m} \mid v_{m}\right\rangle^{2}$ are the FC factors, and $m_{0}$ is the larger of 0 and $-p_{u}$.

Calculation of the $\mathrm{FC}$ factors requires the following parameters: the FC offset $a_{u v}, p_{U}$ and the Manneback angle $\theta$, a measure of the ratio of parabola force constants defined as:

$$
\operatorname{tg}^{2} \theta=\hbar \omega_{v} / \hbar \omega_{u}
$$

Considering two electronic states $u$ and $v$ the luminescence efficiency $\eta_{u v}$ can be expressed in terms of $R$ and $N$ :

$$
\eta_{u v}=\frac{R}{R+N}=\frac{R_{u v}}{R_{u v}+N_{u v} U_{p_{U}}} .
$$

For our hypothetical phosphor $R_{u v}$ and $N_{u v}$ are taken to be $10^{4}$ and $10^{14} \mathrm{sec}^{-1}$, respectively. Equation (4) then reduces to:

$$
\eta_{u v}=\left[1+10^{10} U_{p_{U}}\right]^{-1} .
$$

A computer program was employed to calculate $U_{p_{U}}$. First the linear coupling case was used (i.e., $\theta=45^{\circ}$ ). The phonon frequencies in ground and excited states $\hbar \omega_{0}$ were chosen to be $500 \mathrm{~cm}^{-1}$. The input parameters $a_{u v}$ and $p_{U}$ were obtained from the spectra of the hypothetical phosphor (Fig. 2). The results of the QMSCC calculations using the starting data are presented in Table I and Fig. 3.

We also performed QMSCC calculations after variation of the $U_{p_{U}}$ input parameters. First we varied the zero phonon energy $E_{z p}$, i.e., we induced a vertical displacement of the upper $v$ parabola, (Fig. 1) resulting in an overall shift of the absorption- and emission spectrum (Fig. 2). The results of these calculations are presented in Table I and Fig. 3a.

Appropriate phonon energies for QMSCC calculations are hard to determine. Phononenergies can be evaluated using the temperature dependent shapes of emission and absorption spectra, but values obtained in this way fail to give satisfying results (see,

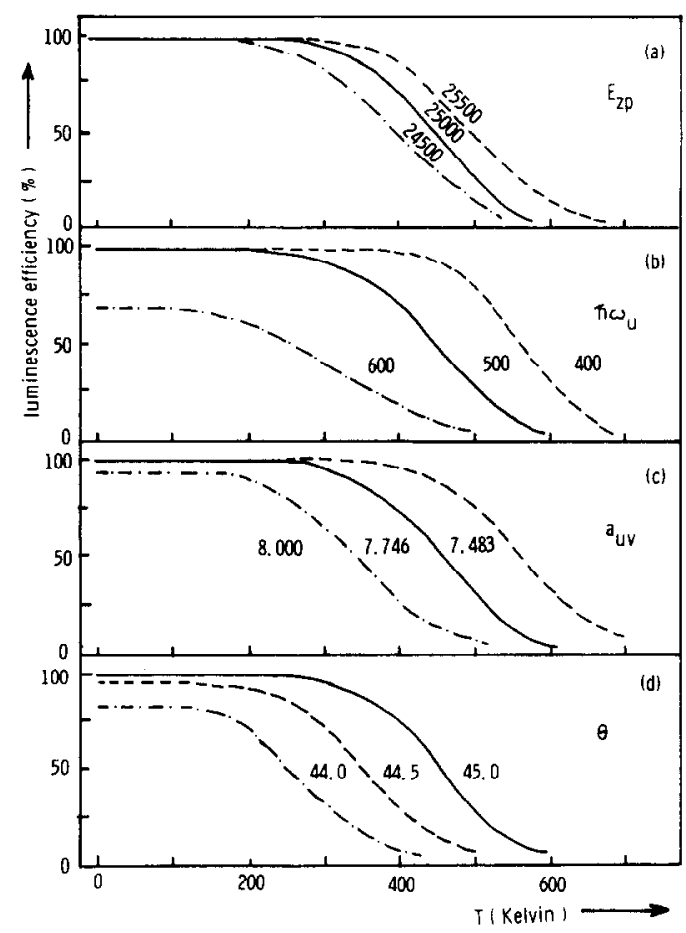

FIG. 3. Temperature dependence of the luminescence intensity of various model phosphor systems.

e.g., Ref. (4)). For our model phosphor we have chosen $\hbar \omega_{u}$ to be 400,500 , and $600 \mathrm{~cm}^{-1}$. Using these data we obtained $a_{u v}$ and $p_{U}$ from the data of Fig. 2 for $\theta=45^{\circ}$. Luminescence efficiency calculations for this system are presented in Table I and Fig. $3 \mathrm{~b}$.

The third parameter we varied was $a_{u v}$. The offset was chosen in such a way that the absorption maximum was shifted $500 \mathrm{~cm}^{-1}$ to lower or $500 \mathrm{~cm}^{-1}$ to higher energy in comparison with the $32,500-\mathrm{cm}^{-1}$ excitation maximum in the starting case. The results of QMSCC calculations performed on this system are presented in Table I and Fig. 3c. Finally we varied the Manneback angle $\theta$. For $\theta \neq 45^{\circ}$ this means there is a difference between the $u$ - and $v$-parabola force constants, i.e., $\hbar \omega_{v} \neq \hbar \omega_{u}$. Table I and Fig. 3d give the results of luminescence efficiency calculations that have been performed on this system. 


\section{Discussion}

Figure $3 \mathrm{a}$ gives the temperature dependence of the luminescence efficiency for different values of the zero phonon energy $E_{\mathrm{zp}}$. For higher values of $E_{\mathrm{zp}}$ the luminescence quenches at higher temperatures. Our results suggest a linear relation between zero phonon energy and quenching temperature. This agrees with a relation found experimentally before for the octahedral niobate and tungstate group ( 7$)$.

As can be seen from Table. I and Fig. 3c small variations in the Franck-Condon offset influence the luminescence efficiency drastically. The offset between the two parabolas is strongly influenced by the bond strength of the surroundings of the luminescent ion. The variations we used here can easily be expected for one and the same luminescing ion in different host lattices. Uranium-doped perovskite host lattices provide good systems to illustrate this phenomenon $(8)$. The quenching temperature of the uranate luminescence in these lattices depends on the nature of the cation between the oxygen ions surrounding the hexavalent uranium ion. If they are small, they will restrict expansion of the uranate group after excitation; i.e., the offset is relatively small. More detailed studies of the temperature quenching of uranate luminescence in perovskite host lattices using QMSCC calculations are the subject of present investigations.

The results of our calculations indicate that lattices with low vibrational frequencies provide suitable hosts for efficient luminescent materials (Table I, Fig. 3b). Note that for $\hbar \omega_{u}=600 \mathrm{~cm}^{-1}$ the quantum efficiency remains far below $100 \%$ even at low temperatures. This result explains why alkali halides with much weaker bonding than in oxides are often suitable hosts for luminescing ions. The phonon energy that should be used in QMSCC calculations is an average frequency. Its determination,

TABLE I

Results of QMSCC CALCUlations ON MOdel PhOSPHORs ${ }^{a}$

\begin{tabular}{|c|c|c|c|c|c|c|c|}
\hline Variable & & $E_{\mathrm{zp}}\left(\mathrm{cm}^{-1}\right)$ & $\hbar \omega_{u}\left(\mathrm{~cm}^{-1}\right)$ & $\theta$ & $a_{u v}$ & $p_{u}$ & $T_{1 / 2}(\mathrm{~K})$ \\
\hline & $x$ & 25,000 & 500 & 45.0 & 7.746 & 50.00 & 450 \\
\hline \multirow{3}{*}{$E_{\mathrm{zp}}$} & & 25,500 & & & & 51.00 & 400 \\
\hline & $x$ & 25,000 & & & & 50.00 & 450 \\
\hline & & 24,500 & & & & 49.00 & 500 \\
\hline \multirow{3}{*}{$\hbar \omega_{u}$} & & & 400 & & 8.660 & 62.50 & 560 \\
\hline & $x$ & & 500 & & 7.746 & 50.00 & 450 \\
\hline & & & 600 & & 7.071 & 41.67 & 325 \\
\hline \multirow{3}{*}{$a_{u v}$} & & & & & 8.000 & & 350 \\
\hline & $x$ & & & & 7.746 & & 450 \\
\hline & & & & & 7.483 & & 560 \\
\hline \multirow{3}{*}{$\theta$} & & 25,000 & & 45.0 & 7.746 & 50.00 & 450 \\
\hline & $x$ & 25,260 & & 44.5 & 7.812 & 50.52 & 360 \\
\hline & & 25,525 & & 44.0 & 7.875 & 51.05 & 285 \\
\hline
\end{tabular}

${ }^{a}$ The data presented in the $\times$-marked rows were used as a starting point. Only parameters differing from the ones in the first row have been enumerated. $T_{1 / 2}$ is defined as the temperature at which the luminescence efficiency has decreased to half of its maximum value. 
however, is a hard task. In practice this parameter is often used as a fit parameter $(4-6)$.

Variation of the Manneback angle $\theta$ also influences the temperature quenching of luminescence (Table I, Fig. 3d). For luminescent materials $\theta$ can be determined using the temperature dependent shape of emission and excitation spectra or directly from the vibrational fine structure in the luminescent spectra.

For a luminescent ion in different host lattices the variations described here will occur neither separately nor independently. Especially the relation between $a_{u v}$ and $\theta$ is obvious.

Although we used a rough approximation to describe radiative and radiationless processes in luminescent materials it is interesting to see that we could get information about the influence of QMSCC parameters on quenching temperature and luminescence efficiency, and that we could get an approximate idea of the nature of the factors influencing the luminescence efficiency. We feel that the results of the present calculations can be used as rules of thumb in predicting and explaining efficient luminescent materials.

In conclusion we note that the parameters used in the case of our hypothetical phosphor seem to be rather extreme. It is necessary, for example, to use a large Stokes shift (large $a_{u v}$ ) for a model phosphor emitting in the visible range of the spectrum, having an average phonon frequency of $500 \mathrm{~cm}^{-1}$, in order to obtain a reasonable quenching temperature (Fig. 2). This may indicate that the present calculations are either inadequate or that more than one excited state is involved in the quenching process (compare Ref. (6)). We will return to this point later. For the present results, the absolute values of the parameters are not very relevant in view of our interest in their variations.

\section{Acknowledgments}

Dr. C. W. Struck of R.C.A. Laboratories, Princeton, Ncw Jerscy, is gratefully acknowledged for furnishing the ASF 100 computer program. The authors are much indebted to Dr. A. B. van Oosterhout, who converted the ASF 100 program to Fortran IV extended for the Control Data 6500 .

\section{References}

1. C. W. Struck and W. H. Fonger, $J$. Luminesc. 10, 1 (1975).

2. E. U. Condon, Phys. Rev. 32, 858 (1928).

3. C. Manneback, Physica 17, 1001 (1951).

4. W. H. Fonger and C. W. STruck, Phys. Rev. B 11, 3251 (1975).

5. C. E. Tyner and H. G. Drickamer, $J$. Chem. Phys. 67, 4103 (1977).

6. C. E. TyNer AND H. G. Drickamer, J. Chem. Phys. 67, 4116 (1977).

7. G. Blasse, J. Chem. Phys. 48, 3108 (1968); G. Blasse AND A. BRIL, J. Solid State Chem. 2, 291 (1970).

8. J. TH. W. DE Hair ANd G. Blasse, J. Solid State Chem. 19, 263 (1976). 